\title{
A Survey on Objectifies of Clustering With Different Strategies
}

\author{
V. Srikanth 1), Weichang Zhang2)
}

\begin{abstract}
Bunching is imperative in information examination and information mining applications. There are diverse kinds of bunches: Well-isolated groups, Center-based groups, Contiguous groups, Density-based bunches, Shared Property or Conceptual Clusters. Prescient and the clear are the two primary errands of the information mining. Grouping should be possible by the diverse no. of calculations, for example, various leveled, parceling, lattice and thickness based calculations. Progressive grouping is the availability based bunching. Apportioning is the centroid-based grouping, the estimation of k-mean is set. Thickness based bunches are characterized as a territory of higher thickness than the staying of the informational index. Network-based bunching is the quickest preparing time that regularly relies upon the extent of the matrix rather than the information. The framework based techniques utilise the single uniform network work to segment the whole issue area into cells. In this overview paper, a survey of grouping and its distinctive procedures in information mining is finished.
\end{abstract}

Keywords: Clustering, Types of Clustering, Classification, Bunch, Information, Mining

\section{Introduction}

Information mining comprises of concentrate, change and load exchange information onto the information stockroom framework, Store and deal with the information in a multidimensional database framework, Provide information access to business experts and data innovation experts, Analyze the information by application programming, Present the information in a helpful configuration, for example, a chart or table. Information mining includes the peculiarity discovery; affiliation administers learning, grouping, relapse, outline and bunching. In this paper, grouping examination is finished. Group Analysis, a programmed procedure to discover similar items from a database. It is a principal activity in information mining[1].

Another sort of bunching is calculated grouping: at least two articles have a place with a similar group if this one characterizes an idea usual to every one of that items. As it were, objects are assembled by their fit to clear ideas, not as per straightforward similitude measures.

Received(September 10, 2019), Review Result(1st: September 30, 2019, 2nd: November 8, 2019), Accepted(December 23, 2019)

1) (Professor) Department of CSE, Pydah College of Engineering and Technology, Visakhapatnam, AP, India email: santhasri99@gmail.com

2) (Professor, Corresponding Author) College of Computer and Information, Hohai University, China email: zhangwc52139@foxmail.com 


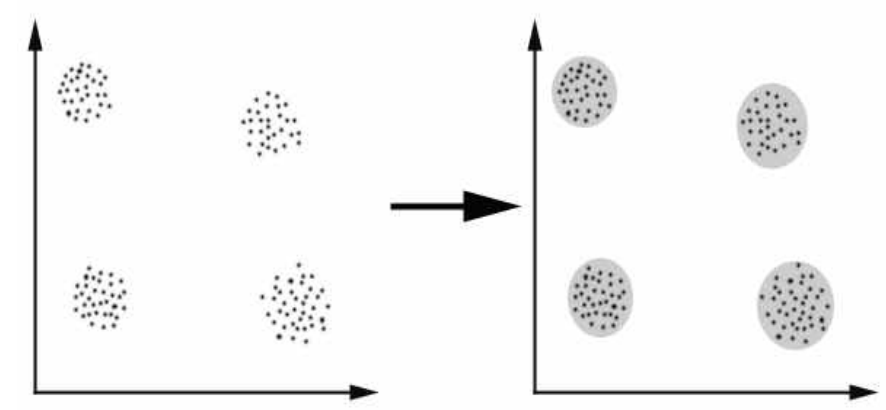

[Fig. 1] Demonstration of Clustering with a straightforward graphical illustration[1]

\section{The Objectives of Clustering}

In this way, the objective of bunching is to decide the inherent gathering in an arrangement of unlabeled information. Be that as it may, how to choose what constitutes a decent bunching? It can be demonstrated that there is no outright "best" model which would be free of the last point of the bunching[1][2].

\section{Applications, Necessities And Issues of A Clustering}

\subsection{Conceivable Applications}

Grouping calculations can be connected in numerous fields, for example:

- Marketing: discovering gatherings of clients with comparable conduct given a vast database of client information containing their properties and past purchasing records

- Biology: arrangement of plants and creatures given their highlights

- Libraries: book requesting

- Insurance: distinguishing gatherings of engine protection arrangement holders with a high standard claim cost; recognizing cheats

- City-arranging: distinguishing gatherings of houses as per their home kind, esteem and geological area

- Earthquake examines: bunching watched tremor epicenters to recognize hazardous zones

-WWW: record order; bunching weblog information to find gatherings of comparable access designs[3][4][5].

\subsection{Necessities}


a. scalability

b. dealing with various sorts of qualities

c. discovering groups with discretionary shape

d. minimal necessities for space learning to decide input parameters

e. ability to manage clamour and anomalies

f. insensitivity to a request for info records

g. high dimensionality

h. interpretability and ease of use

\subsection{Issues}

There are various issues with grouping. Among them:

- current grouping methods do not address every one of the prerequisites enough (and simultaneously)[3]

- dealing with a massive number of measurements and the vast number of information things can be dangerous as a result of time multifaceted nature

- the viability of the technique relies upon the meaning of "remove" (for separate based bunching)

- if a conspicuous separation measure does not exist we should "characterize" it, which isn't generally simple, particularly in multi-dimensional spaces

- the aftereffect of the grouping calculation (that much of the time can be self-assertive itself) can be deciphered in various ways[4][6].

Data Mining is a four-stage: Assemble information, Apply information mining apparatuses on datasets, Interpretation and assessment of result, Result application.

Exhibiting information by fewer groups permanently loses specific excellent points of interest (misfortune in information pressure), however, accomplishes rearrangements. It speaks to numerous information protests by few groups, and subsequently, it demonstrates information by its bunches. Bunching is frequently one of the initial phases in information mining investigation. It distinguishes gatherings of related records that can be utilised as a beginning stage for investigating further connections. Bunching is an information mining (machine learning) system used to put information components into related gatherings without propel information of the gathering definitions. Bunching strategies fall into a gathering of undirected information mining devices. The objective of undirected information mining is to find structure 
in the information all in all. All in all, there are two sorts of qualities related to input information in bunching calculations, i.e., numerical characteristics, and straight out properties. Numerical characteristics are those with a limited or endless number of requested qualities, for example, the stature of a man or the $x$-arrange of a point on a $2 \mathrm{D}$ area. Then again, downright characteristics are those with limited unordered qualities, for example, the occupation or the blood classification of a man[5][6].

\section{General Types of Clusters}

\subsection{Very Much Isolated Groups}

A bunch is an arrangement of focuses with the goal that any point in a bunch is closest (or more comparative) to each other point in the bunch when contrasted with whatever another point that isn't in the bunch.

\subsection{Focus-Based Bunches}

A group is an arrangement of items with the end goal that a question in a bunch is closest (more comparative) to the "middle" of a group than to the focal point of some other bunch. The focal point of a group is regularly a centroid.

\subsection{Touching Bunches}

A group is an arrangement of focuses with the goal that a point in a bunch is closest (or more comparative) to at least one different focuses in the group when contrasted with any point that isn't in the bunch.

\subsection{Thickness Based Groups}

A bunch is a thick district of focuses, which is isolated by as per the low-thickness locales, from different areas that are of high thickness.

\subsection{Shared Property or Conceptual Clusters}

Finds groups that offer some common property or speak to a specific idea[7]. 


\section{Information Mining Tasks}

Data mining errands are for the most part isolated into two noteworthy classes:

\subsection{Predictive Errand}

The objective of this assignment is to foresee the estimation of one specific trait, in light of estimations of different qualities. The traits that are utilized for making the expectation is named as an independent variable. The other esteem which is to be anticipated is known as the Target or ward esteem.

\subsection{Descriptive Errand}

The reason for this assignment is to gather essential relations in information. In elucidating undertaking of information mining, values are autonomous, and it habitually requires present preparing for approving comes about.

\section{Various Clustering Algorithms}

Grouping oF Clustering is the primary assignment of Data Mining. What's more, it is finished with the number of calculations. The most generally utilised calculations in Clustering are Hierarchical, Partitioning, Density and Grid-based calculations.

\subsection{Various Levelled / Hierarchical Algorithms}

The various leveled grouping includes making bunches that have a foreordained requesting start to finish. For instance, all records and envelopes on the hard plate are sorted out in order. There are two sorts of progressive grouping, Divisive and Agglomerative.

\section{Troublesome Strategy}

In troublesome or top-down grouping strategy we appoint the majority of the perceptions to a single bunch and afterwards segment the group to two slightest similar groups. At long last, we continue recursively on each bunch until there is one group for every perception. There is proof that disruptive calculations deliver more precise progressive systems than agglomerative calculations in a few conditions, however, is theoretically more mind-boggling. 


\section{Agglomerative Technique}

In agglomerative or base up bunching strategy, we allocate every perception to its group. At that point, process the similitude (e.g., remove) between every one of the bunches and join the two most similar groups. At long last, rehash stages 2 and three until there is just a solitary bunch left

\subsubsection{Advantages Of Various Levelled Grouping}

1. Inserted adaptability concerning the level of granularity.

2. The simplicity of taking care of any types of comparability or separation.

3. Relevance to any properties write.

\subsubsection{Disadvantages Of Various Levelled Grouping}

1. The ambiguity of end criteria.

2. The most hierarchal calculation does not return to once developed groups with the motivation behind the change.

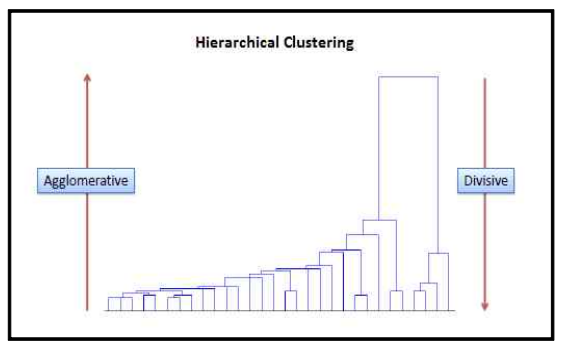

[Fig. 2] Various leveled / Hierarchical Algorithms[7]

\subsection{Partitioning Algorithms}

Apportioning bunching is bunching strategies used to arrange perceptions, inside an informational collection, into different gatherings in light of their closeness. The calculations require the expert to indicate the number of bunches to be produced.

This section depicts the customarily utilized dividing grouping, including:

- K-implies bunching (MacQueen 1967), in which, each group is spoken to by the inside or methods for the information directs having a place toward the bunch. The K-implies technique is touchy to bizarre information focuses and exceptions.

- K-medoids bunching or PAM (Partitioning Around Medoids, Kaufman and Rousseeuw, 1990), in which, each group is spoken to by one of the articles in the group. PAM is less 
delicate to anomalies contrasted with k-implies.

- CLARA calculation (Clustering Large Applications), which is an augmentation to PAM adjusted for extensive informational collections.

For every one of these techniques, we give:

- The essential thought and the critical scientific ideas

- The grouping calculation and usage in $\mathrm{R}$ programming

- $\mathrm{R}$ lab segments with numerous cases for bunch investigation and perception

The accompanying $\mathrm{R}$ bundles will be utilized to register and imagine parceling grouping:

- Stats bundle for registering K-implies

- Cluster bundle for processing PAM and CLARA calculations

- Fact extra for delightful representation of bunches

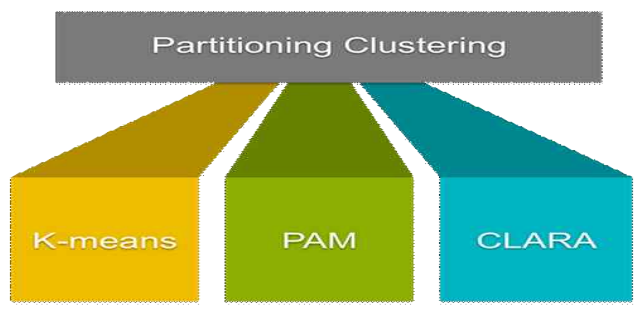

[Fig. 3] Partitioning Clustering[1][2]

The k-implies calculation is the most prominent grouping device that is utilized as a part of consistent and mechanical applications.

The essential calculation is elementary:

1. Select $\mathrm{K}$ focuses as initial centroid.

2. Rehash.

3. Shape $\mathrm{K}$ bunches by relegating each point to its nearest centroid.

4. Recomputed the centroid of each bunch until the point that centroid does not change.

The k-implies calculation has the accompanying essential properties:

1. It is productive in handling extensive informational collections.

2. It frequently ends at a neighborhood ideal.

3. It works just on numeric qualities.

4. The groups have raised shapes.

\subsection{Density-Based Clustering}


The Density-based Clustering device works by recognizing zones where focuses are concentrated and where they are isolated by territories that are vacant or scanty. Focuses that are not some portion of a bunch are named as commotion.

This device utilizes unsupervised machine getting the hang of grouping calculations which consequently distinguish designs construct concerning spatial area and the separation to a predefined number of neighbors. These calculations are viewed as unsupervised because they do not require any preparation on being a bunch.

Thickness based bunching calculation has assumed an indispensable part in discovering non-direct shapes structure in light of the thickness. Thickness Based Spatial Clustering of Applications with Noise (DBSCAN) is most generally utilized thickness based calculation. It utilizes the idea of thickness reach ability and thickness network.

This is tying process. Along these lines, if "q" is a neighbor of "r", "r" is a neighbor of "s", "s" is a neighbor of " $t$ " which like this is a neighbor of "p" infers that "q" is a neighbor of "p".

\section{Algorithmic Strides for DBSCAN Grouping}

Let $X=\{x 1, x 2, x 3, \ldots, x n\}$ be the arrangement of information focuses. DBSCAN requires two parameters: $\varepsilon$ (epsilon) and the base number of focuses required to frame a bunch (minPts).

1) Start with a subjective beginning stage that has not been gone by.

2) Extract the area of this point utilizing $\varepsilon$ (All focuses which are inside the $\varepsilon$ separate are neighborhood).

3) If there are adequate neighborhood around this point at that point bunching process begins, and the point is set apart as went by else this point is marked as clamour (Later this point can turn into the piece of the group).

4) If a point is observed to be a piece of the group, then its $\varepsilon$ neighborhood is likewise the piece of the bunch, and the above strategy from stage 2 is rehashed for all $\varepsilon$ neighborhood focuses. This is rehashed to the point when all focus on the bunch is resolved.

5) another unvisited point is recovered and handled, prompting the revelation of a further group or commotion.

6) This procedure proceeds until the point that all focuses are set apart as went to.

\section{Points of Interest}

1) Does not require from the earlier detail of some groups.

2) Able to recognize commotion information while grouping.

3) DBSCAN calculation can discover self-assertively estimate and subjectively moulded 
bunches.

\section{Detriments}

1) DBSCAN calculation flops if there should arise an occurrence of changing thickness bunches.

2) Fails in the event of neck kind of dataset.

3) Does not function admirably if there should be an occurrence of high dimensional information[7].

\section{Potential Applications}

A portion of the ways this apparatus may be connected are as per the following:

- Urban water supply systems are a critical shrouded underground resource. The bunching of pipe breaks and blasting can demonstrate approaching issues. Utilizing the Density-based Clustering instrument, an architect can discover where these groups are and make a pre-emptive move on high-risk zones inside water supply systems.

- Suppose you have position information for all fruitful and unsuccessful shots for NBA players. Thickness based Clustering can demonstrate to you the notable examples of fruitful versus fizzled shot positions for every player.

- Say you are examining a specific vermin borne ailment and have a point dataset speaking to family units in your investigation territory, some of which are swarmed, some of which are not. By utilizing the Density-based Clustering instrument, you can decide the biggest bunches of plagued family units to help pinpoint a territory to start treatment and eradication of bugs.

- Geo-finding tweets following characteristic dangers or fear assaults can be bunched and save, and departure needs can be educated in light of the size and area of the groups recognized.

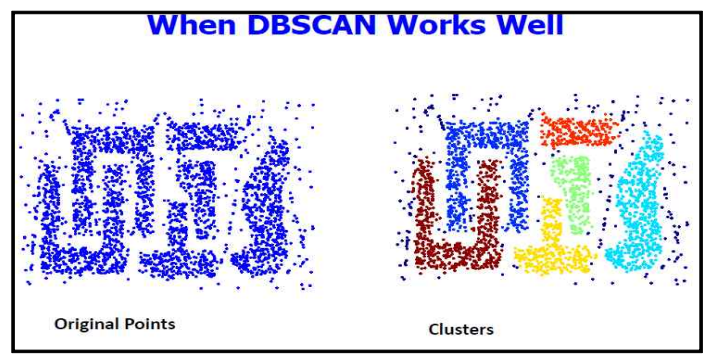

[Fig. 4] Working Principal of DBSCAN[7]

\subsection{Grid Based Algorithms}


Lattice-based grouping where the information space is quantized into a limited number of cells which shape the matrix structure and perform bunching on the frameworks. Framework-based bunching maps the limitless number of information records in information streams to limited quantities of lattices. Lattice-based bunching is the quickest handling time that commonly relies upon the measure of the network rather than the information. The lattice-based strategies utilise the single uniform network work to parcel the whole issue area into cells, and the information objects situated inside a cell are spoken to by cell utilizing an arrangement of measurable qualities from the articles. These calculations have a quick handling time since they experience the informational index once to register the measurable qualities for the networks and the execution of bunching depends just on the extent of the matrices which is typically significantly less than the information objects. The framework based bunching calculations are STING, Wave Cluster, and CLIQUE. Every one of these techniques utilizes a regular matrix work to cover the entire issue. For the issues with exceptionally sporadic information dissemination, the determination of the matrix work must be too beautiful to get a decent grouping quality. A better work can bring about the work estimate near or even surpass the extent of the information objects, which can use note worthily increment the calculation stack for bunching[7].

Versatile Mesh Refinement Adaptive Mesh Refinement (AMR) is a sort of multi resolution calculation. This calculation accomplishes high determination in limited areas of dynamic, multi-dimensional numerical reenactments. This is effectively connected to display colossal scale logical applications in the scope of orders, for example, computational liquid elements, astronomy, meteorological reproductions, auxiliary progression, attractive, and warm elements. Necessarily, it can put great determination lattices accurately where the high computational cost requires. The versatility of the calculation permits mimicking multi resolution that are distant with techniques utilizing a worldwide uniform beautiful lattice. The AMR grouping calculation right off the bat makes various determination frameworks in light of the thickness. After that frameworks include a pecking order tree that speaks to the issue space as settled organized matrices of expanding determination. The calculation considers each leaf as the focal point of an individual bunch and recursively doles out the enrollment for the information objects situated in the parent hubs until the point when the root hub is come to. The AMR bunching calculation can identify the settled groups at various levels of resolutions by utilizing the progressive tree. As the AMR calculation is a lattice thickness based calculation, so it likewise shares the essential qualities of all framework based strategies. AMR calculation has a quick preparing time. It can isolate from the clamour. The request of info information is coldhearted. 
AMR is a method that begins with a coarse uniform framework covering the whole computational volume and naturally refines specific locales by including better sublattices. From the associated parent lattice cells, the new youngster networks are made whose properties, the thickness, for example, surpass given edges

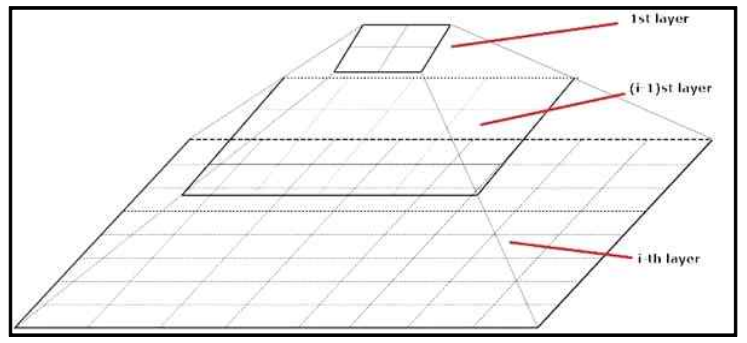

[Fig. 5] Grid-Based Algorithms

\section{Conclusions}

Grouping is critical in information examination and information mining applications. Grouping should be possible by the distinctive no. of calculations, for example, various leveled, dividing, lattice and thickness based calculations. Various leveled grouping is the network-based bunching. Parceling is the centroid-based bunching, the estimation of k-mean is set. Thickness based groups are characterized as a region of higher thickness than the staying of the informational collection. Lattice-based bunching is the speediest preparing time that normally relies upon the span of the matrix rather than the information. The matrix-based techniques utilize the single uniform latticework to segment the whole issue space into cells.

\section{References}

[1] Jui-Chien Hsieh, Ai-Hsien Li and Chung-Chi Yang, Mobile, Cloud, and Big Data Computing: Contributions, Challenges, and New Directions in Telecardiology, International Journal of Environmental Research and Public Health, (2013), Vol.10, No.11, pp.6131-6153, DOI:10.3390/ijerph10116131

[2] Marcos D. Assunção, Rodrigo N. Calheiros, Silvia Bianchi, Marco A.S. Netto, Rajkumar Buyya, Big Data computing and clouds: Trends and future directions, Journal of Parallel and Distributed Computing, (2015), Vol.79-80, No.8, pp.3-15, DOI: https://doi.org/10.1016/j.jpdc.2014.08.003

[3] Sharma, R. and R.K. Trivedi, Literature review: Cloud Computing-Security Issues, Solution and Technologies, International Journal of Engineering Research, (2014), Vol.3, No.4, pp.221-225, DOI: 
$10.17950 /$ ijer/v3s $4 / 408$

[4] Khan, A.W., S.U. Khan, M. Ilyas, and M.I. Azeem, A literature survey on data privacy/protection issues and challenges in cloud computing, IOSR Journal of Computer Engineering (IOSRJCE), (2012), Vol.1, No.3, pp.28-36.

[5] Monisha, Nagalakshmi, Suneetha, study on Big Data, International conference on emerging technologies, networking and computational intelligence, (2016), March 28; Chennai, Tamil Nadu, India

[6] Islam, S. and J.-C. Grégoire, Giving users an edge: A flexible Cloud model and its application for multimedia, Future Generation Computer Systems, (2012), Vol.28, No.6, pp.823-832, DOI: https://doi.org/10.1016/j.future.2012.01.002

[7] Ghanshyam Dewta, Rajesh Tiwari, A Distributed Approach for Development of the DBSCAN Clustering, International Journal of Advance Research, Ideas and Innovations in Technology, (2017), Vol.3, No.2, pp.975-979. 